Cite this: J. Anal. At. Spectrom., 2013, 28, 1080

Received 20th February 2013 Accepted 22nd April 2013

DOI: 10.1039/c3ja50059d www.rsc.org/jaas

\title{
A novel ToF-SIMS operation mode for improved accuracy and lateral resolution of oxygen isotope measurements on oxides
}

\author{
Gerald Holzlechner, $\uparrow^{*}$ Markus Kubicek, $\uparrow$ Herbert Hutter and Jürgen Fleig \\ Oxygen isotope exchange with subsequent time-of-flight secondary ion mass spectrometry (ToF-SIMS) is a \\ highly valuable tool for determining oxygen diffusion coefficients in oxides. Since ToF-SIMS analysis enables \\ an elemental and chemical mapping, it can also be used to visualize oxygen exchange-active zones by \\ determining the local oxygen isotopic fraction. However, measuring accurate isotopic fractions can be a \\ challenging analytical task owing to secondary ion interaction and signal saturation, particularly when \\ dealing with high secondary ion intensities as commonly found when analyzing oxygen ions from \\ oxides. It is shown that in many cases the calculated ${ }^{18} \mathrm{O}^{-}$fraction erroneously shifts to higher values \\ and can lead to systematic errors in the determination of diffusion coefficients. A novel ToF-SIMS \\ operation mode, called "Collimated Burst Alignment" (CBA) mode, is therefore introduced to enable a \\ more accurate determination of oxygen isotopic fractions with an optimized lateral resolution of sub- \\ $100 \mathrm{~nm}$. Both improvements are rendered possible by a modified beam guidance in the primary ion \\ gun. This modification reduces detector dead time effects and ion interactions to a minimum and \\ secondary ion intensities can be obtained more accurately. The result of this optimization is \\ demonstrated in measurements of the natural isotope abundance of several different oxides including \\ $\mathrm{SrTiO}_{3}$ and $\mathrm{Sr}$-doped $\mathrm{LaCoO}_{3}$.
}

\section{Introduction}

Functional oxides find application in many different fields, ranging from solid oxide fuel cells (SOFCs) to ferroelectric memories, piezoelectric actuators and gas sensors. In most fields the oxide ion motion plays a role either in preparation, operation or degradation. For example, the ion transport in solid electrolytes or the oxygen reduction at cathodes crucially affects the cell efficiency of SOFCs. ${ }^{1-33}$ In ferro- and piezoelectric oxides degradation phenomena such as an increasing leakage current upon DC load or fatigue are often related to oxide ion motion..$^{34-50}$

A powerful tool to quantify oxide ion diffusion and to visualize electrochemically active zones of oxides is the combination of tracer oxygen incorporation with subsequent secondary ion mass spectrometry (SIMS) analysis., ${ }^{4,6,8,9,11,13-15,17,19,20,22,26-33,43,45-47,51-55}$ By using time-of-flight- (ToF-) SIMS, detailed 2D and 3D information about the chemical composition of a surface and the elemental distribution can then be obtained. ${ }^{56,57}$ Elemental or isotope ion ratios can be determined for quantitative data interpretation. ${ }^{58,59}$ Among others, oxygen tracer diffusion

Institute of Chemical Technologies and Analytics, Vienna University of Technology, Vienna, Austria. E-mail: gerald.holzlechner@tuwien.ac.at; Fax: +43 158801 15899; Tel: +4315880115821

$\dagger$ Authors with equal contribution. coefficients and surface exchange coefficients can therefore be evaluated from ToF-SIMS depth profiles of the local oxygen isotopic fraction. ${ }^{60}$

The main challenge when measuring isotopic fractions is to achieve sufficient ion intensities for minor isotopes while preventing the major isotope signals from saturation..$^{58}$ This is far from trivial since SIMS analysis of oxygen in oxides can result in high negative secondary ion intensities due to an enhanced oxygen ionization yield and high primary ion intensities in established ToF-SIMS modes. High secondary ion intensities can cause significant collision-induced ion interaction $\mathrm{s}^{58,61}$ and detector dead time effects, ${ }^{62-64}$ both leading to an underestimated value for the detected major isotope intensities. Consequently, high secondary ion intensities can lead to systematic errors in the quantitative data interpretation, as detailed in this paper. One approach to minimize the error of oxygen isotopic fractions is described in the literature by De Souza et al. ${ }^{61}$ Operating ToF-SIMS in BA-burst mode reduces the intensity per primary ion pulse by chopping several pulses (bursts) out of a larger one. The determination of accurate diffusion profiles in BA-burst mode, however, requires an elaborate analysis of the raw data. Moreover, the lateral resolution is reduced. As an indication for an accurate measurement of the isotopic fraction the ${ }^{18} \mathrm{O}$ level of oxides can be measured after equilibration in ambient air and compared with the 
expected natural abundance value. This corresponds to the test of the accuracy of a SIMS operation mode in that part of a tracer concentration profile where the major isotope intensity (here $I_{160}$ ) is at its maximum and shows the greatest impact on the error of the isotopic fraction.

In this contribution, we first show that measurements of ${ }^{18} \mathrm{O}$ intensities in several oxides such as $\mathrm{SrTiO}_{3}, \mathrm{MgO}$ and $\mathrm{Sr}$-doped $\mathrm{LaCoO}_{3-\delta}$ consistently show an erroneously high fraction when using ToF-SIMS analysis in established "Burst Alignment" (BA) and "High Current Bunched" (HCBU) mode, despite varying primary ion emission current and cluster size. The aim was therefore to develop an optimized ToF-SIMS mode in order to improve this situation by shifting secondary ion intensities to an optimal level to guarantee an accurate determination of both the major $\left({ }^{16} \mathrm{O}^{-}\right)$and minor $\left({ }^{18} \mathrm{O}^{-}\right)$secondary ion intensities. These developments were performed on an ION-TOF instrument but all basic ideas can also be applied to other instruments. It is shown that a novel mode called "Collimated Burst Alignment" (CBA) mode indeed leads to highly accurate tracer fraction values. Simultaneously it enables an improved lateral resolution of sub-100 $\mathrm{nm}$ compared to $\sim 250$ to $300 \mathrm{~nm}$ in BA or BA-burst mode. Moreover, the CBA mode gives the possibility of increasing the mass resolution to $m / \Delta m>6000$ by bursting the primary ion beam - thus being able to differentiate between secondary ions of the same mass number, e.g. ${ }^{16} \mathrm{O}_{2}$ and ${ }^{32} \mathrm{~S}$, as we detailed in ref. 65. Reasons for the improved performance of the CBA mode are discussed by detailed analysis of secondary ion mass peaks and effects of dead time and ion interaction.

\section{Experimental}

All SIMS measurements were performed on a TOF.SIMS 5 instrument (ION-TOF, Münster, Germany) using $25 \mathrm{kV} \mathrm{Bi}{ }_{x}{ }^{{ }^{+}}$ primary ions. The ${ }^{18} \mathrm{O}^{-}$fractions were determined by operating the instrument in the novel "Collimated Burst Alignment" (CBA) mode as well as in the conventional modes commonly called "Burst Alignment" (BA) and "High Current Bunched" (HCBU) mode. During operation the primary ion gun typically scans a field of view of $100 \times 100 \mu^{2}$ applying a $1024 \times 1024$ pixel measurement raster. For sequential sputtering a $2 \mathrm{kV} \mathrm{Cs}^{+}$sputter gun $\left(400 \times 400 \mu \mathrm{m}^{2}\right.$ sputter area, $2 \mathrm{~s}$ sputtering, $1 \mathrm{~s}$ pause time $)$ was used. Charge compensation was achieved by $20 \mathrm{eV}$ electrons. ${ }^{\mathbf{6 6 , 6 7}}$

Once the primary ion gun is aligned, a ToF-SIMS mass spectrum is generated by summing the detected secondary ion intensities and plotting them against the mass channels. The mass peak integration limit was set to the peak center plus/ minus half the dead time of $\tau=65.4 \mathrm{~ns}$. Since the primary ion peak width was adjusted to $50 \mathrm{~ns}$ FWHM, the $50 \mathrm{~ns}$ of the main secondary ion mass peak as well as $7.7 \mathrm{~ns}$ before and after this main peak were considered in the integration. Since preceding ion counts can saturate the detector even before ions of the actual mass peak impinge, underestimated intensity counts could have a greater impact to the entire counts of a $\sim 1$ ns pulse than to a longer one. When a single secondary ion peak width is smaller than the detector's dead time (pulse width $\leq \tau$ ), the intensity correction using Poissonian contribution simplifies to the equation ${ }^{58}$

$$
\frac{I_{x_{i}}}{N}=-\ln \left(1-\frac{I_{x_{i}, \text { exp }}}{N}\right),
$$

where $I_{x_{i}, \exp }$ is the integrated measured intensity of ion species $x_{i}, I_{x_{i}}$ the corrected intensity and $N$ the total number of primary ion ionization pulses. The ${ }^{18} \mathrm{O}$ fraction $f_{180}^{*}$ is given by ${ }^{16} \mathrm{O}$ and ${ }^{18} \mathrm{O}$ secondary ion intensities $\left(I_{160}, I_{180}\right)$ and according to

$$
f_{18 \mathrm{O}}^{*}=\frac{I_{18 \mathrm{O}}-I_{18 \mathrm{O}, \mathrm{bg}}}{I_{16 \mathrm{O}}-I_{16 \mathrm{O}, \mathrm{bg}}+I_{18 \mathrm{O}}-I_{18 \mathrm{O}, \mathrm{bg}}}
$$

where $I_{160 \text {,bg }}$ and $I_{180, \text { bg }}$ are the corrected background intensities near the peak.

The lateral resolution was determined by imaging a part of the certified reference material BAM-L200 (ref. 68) as suggested in ref. 69. The reference sample consisted of AlGaAs/GaAs gratings with finely grated periods from $6.9 \mathrm{~nm}$ to $587 \mathrm{~nm}$. Mass spectra, the ${ }^{18} \mathrm{O}$ natural abundance level and details on oxygen isotope mass peaks were obtained on a number of functional oxides. The properties of these oxides range from high electronic and high ionic conductivity $\left(\left(\mathrm{La}_{0.6} \mathrm{Sr}_{0.4}\right) \mathrm{CoO}_{3-\delta},\left(\mathrm{La}_{0.6} \mathrm{Sr}_{0.4}\right)\right.$ $\mathrm{FeO}_{3-\delta}$ ) to moderate or low electron/hole and ion conduction $\left(\mathrm{SrTiO}_{3}, \mathrm{~Pb}(\mathrm{Zr}, \mathrm{Ti}) \mathrm{O}_{3}\right)$ to almost no electronic but high ionic conductivity $\left((\mathrm{Zr}, \mathrm{Y}) \mathrm{O}_{2-\delta}\right)$ or highly insulating material $(\mathrm{MgO})$. Thin films of $\left(\mathrm{La}_{0.6} \mathrm{Sr}_{0.4}\right) \mathrm{CoO}_{3-\delta}$ (LSC) and $\left(\mathrm{La}_{0.6} \mathrm{Sr}_{0.4}\right) \mathrm{FeO}_{3-\delta}$ (LSF) were produced by pulsed laser deposition (PLD) onto (Zr,Y) $\mathrm{O}_{2-\delta}$ (YSZ) single crystals $\left(9.5 \mathrm{~mol} \% \mathrm{Y}_{2} \mathrm{O}_{3}\right)$. The deposition procedures are described elsewhere. ${ }^{19}$ The ${ }^{18} \mathrm{O}$ fractions in LSC and LSF were obtained immediately after PLD preparation as well as after pre-annealing the samples in an ambient atmosphere for 30 minutes at $600{ }^{\circ} \mathrm{C}$. Commercially available single crystals of undoped $\mathrm{SrTiO}_{3}$ (STO), YSZ and $\mathrm{MgO}$ were investigated as-received (Crystec, Germany). A $\mathrm{Pb}\left(\mathrm{Zr}_{1-x} \mathrm{Ti}_{x}\right) \mathrm{O}_{3}$ (PZT) ceramic sample was supplied by Graz University of Technology (Graz, Austria). For surface polishing of the latter one, diamond suspensions (DiaPro 9 and $3 \mu \mathrm{m}$, Struers, Germany) were used with MD-disk Largo (Struers) on a Planopol-3 Pedemax polishing machine (Struers).

\section{ToF-SIMS measurements: problems and conventional operation modes}

\section{Dead time function and intensity saturation}

Sputtering a target surface with a positive primary ion beam of suitable energy leads to interactions that cause the emission of secondary ions. Immediately after the sputtering process the secondary ions are extracted from the surface into the ToFanalyzer by an electrostatic field. After acceleration, the secondary ions get separated according to their mass to charge ratio. Secondary ions arriving at the detector with either only positive or only negative polarity are counted and their arrival times are recorded. Such a detector consists of a microchannel plate for ion-to-electron conversion, a scintillator for electron-tophoton conversion and finally a photomultiplier for recording impinged ions. ${ }^{58}$ Since ToF-SIMS is equipped with a single ion counting detector, it is impossible to distinguish between multiple ions arriving within a certain time interval $\tau$ denoted as the detector dead time. Hence, all ions arriving at the detector 
simultaneously or within the dead time $\tau$ are counted as just one single ion. The Poissonian correction (see eqn (1)) is often suitable to correct the experimentally obtained counts. However, an accurate correction is particularly troublesome for highly intensive secondary ion mass peaks (e.g. $\mathrm{O}^{-}$on oxides) with almost no "zero" events and pronounced dead time effects.

The theory of single ion counting, dead time effects and Poissonian correction has been discussed in detail by Stephan, Zehnpfenning and Benninghoven, who concluded that in many cases a simple correction according to the Poissonian distribution is sufficient, but for a precise correction the dead time function has to be known in detail. ${ }^{64}$ This dead time function reflects the probability that the detector is not able to detect new ions after a time $\tau$. It was also demonstrated that when being saturated once, the detector is still not fully recovered even after $300 \mathrm{~ns}$. For the sake of illustration we simulated the impact of differently shaped dead time functions (DTFs) to secondary ion intensity mass peaks with a long pulse width of about $5 \tau$ (Fig. 1). All DTFs start near unity detector saturation but differ in their decay characteristics. A simple step function leads to a secondary ion mass peak oscillating with a period of dead time $\tau$ (Fig. 1b). Such oscillations are generally not found in
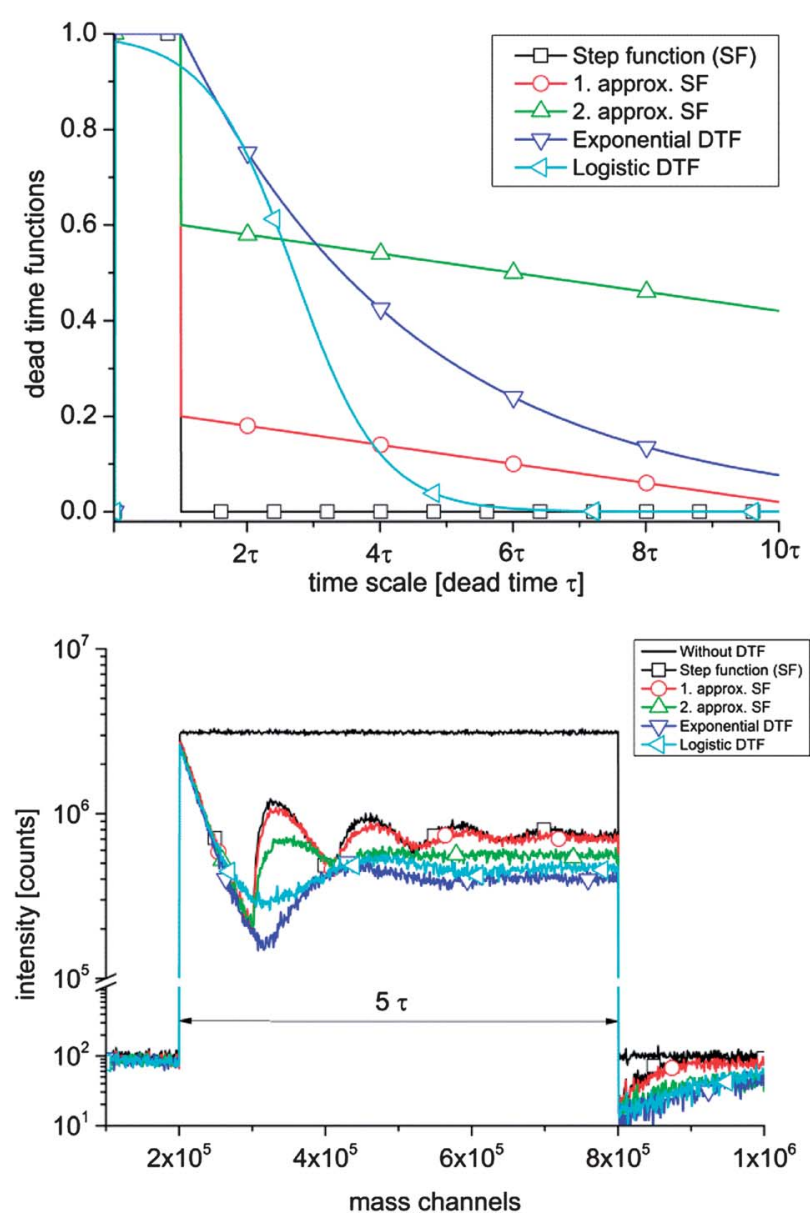

Fig. 1 (a) Differently shaped dead time functions (DTFs) giving the probability that the detector is still dead after the detection of an ion in dead time $\tau$. (b) Impact of the different DTFs to a simulated secondary ion mass spectrum for a $\sim 5$ long pulse.

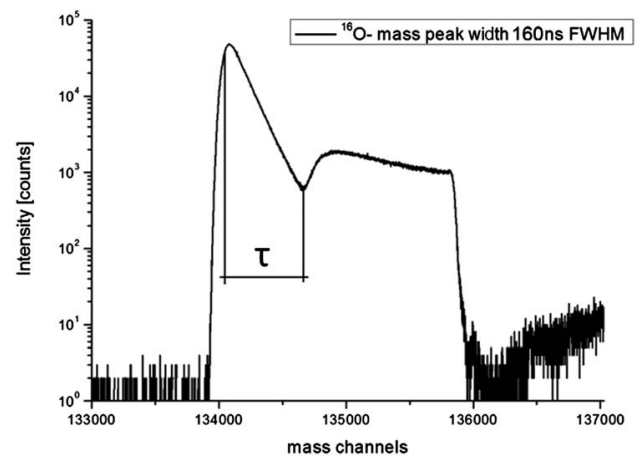

Fig. 2 Experimentally obtained ${ }^{16} \mathrm{O}^{-}$secondary ion mass peak on an as-received $\mathrm{SrTiO}_{3}$ sample in "Burst Alignment" (BA) mode. High secondary ion intensities saturate the single ion counting detector.

measurements and can be reduced by more complex DTFs. For example, an exponential- or logistic-like DTF results in a secondary ion signal, which is in better agreement with typical experimentally obtained secondary ion mass peaks such as the ${ }^{16} \mathrm{O}$ mass peak in Fig. 2. The latter shows a typical example of the dead time impact on a very intensive mass peak (BA mode, $\mathrm{Bi}_{1}^{+}, 0.5 \mathrm{pA}$ pulsed, $160 \mathrm{~ns}$ pulse width). From the time dependence of the saturated intensity signal in Fig. 2 the actual dead time $\tau$ can be determined and found to be $\tau=65.4 \mathrm{~ns}$ fullwidth-half-maximum (FWHM) for our ToF-SIMS setup.

The high oxygen sputter ionization rate together with high primary ion currents in conventional operation modes like "Burst Alignment" or "High Current Bunched" leads to very intensive secondary ion mass peaks in many functional oxides. The main challenge in measuring isotopic fractions is to prevent the major isotope signal from strong saturation of the detector with dead time losses, while achieving sufficient ion intensities for the minor isotope, in order to minimize counting statistical errors. ${ }^{5,61}$ Hence, for an accurate isotopic measurement it is essential to ensure the linearity of the detection system over a wide dynamic range.

\section{"High Current Bunched", "Burst Alignment" and "Collimated" mode}

The primary ion gun can be operated in different modes that mainly differ in their mass resolution, primary ion intensity and achievable lateral resolution. ${ }^{70}$ In the commonly called "High Current Bunched" (HCBU) mode, a bunched and very intensive primary ion beam increases mass resolution $(m / \Delta m>11000)$, however, at the cost of lateral resolution $(2-10 \mu \mathrm{m})$. The "Burst Alignment" (BA) mode is particularly used for fast imaging with moderate mass resolution $(m / \Delta m>200)$ and improved lateral resolution (250-300 $\mathrm{nm})$.

In the BA mode, in the primary ion column two out of three ion optical lenses are energized. After the "Liquid Metal Ion Source" (LMIS) the first lens "Lens Source" positions a beam crossover in the blanking aperture 2, as visualized in Fig. 3. The second lens "Lens Target" is used to focus the primary ion beam to a minimal spot size on the target surface. Relatively high primary ion currents in the range of $1 \mathrm{pA}$ are obtained by running the primary ion gun in pulsed mode $\left(\mathrm{Bi}_{1}{ }^{+}, 100 \mu\right.$ s cycle 


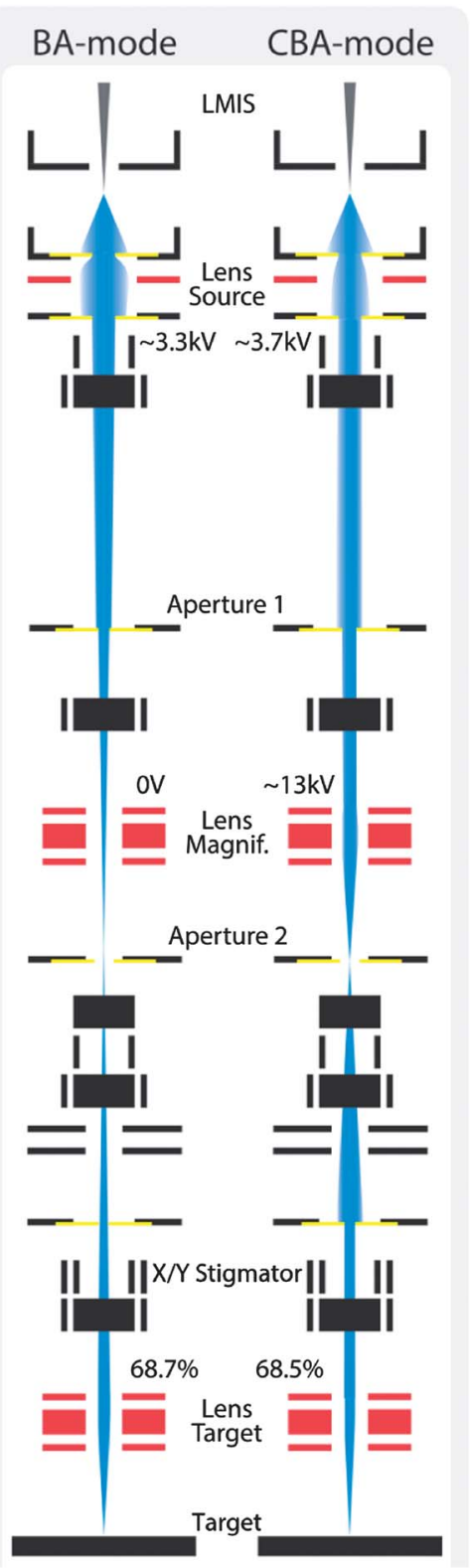

Fig. 3 Primary ion gun scheme of the beam guidance for the BA (Burst Alignment) and CBA (Collimated Burst Alignment) modes on an ION-TOF instrument.

time, 50 ns pulse width). The BA mode is also the starting point to activate the BA-burst mode. The burster activation energizes a sine blanker consisting of a dual set of blanking plates. The socalled chopper cuts a large beam pulse into several smaller ion packages. In this way mass resolution is increased and secondary ion intensity per primary ion pulse is decreased. For very intensive mass peaks such as ${ }^{16} \mathrm{O}^{-}$in oxides the first burst nevertheless saturates the detector, thus affecting the burst following within the detector dead time $\tau$.

Since the dead time function is not precisely known, the Poissonian correction is applied to the first single burst only. To obtain the total ion counts of saturated bursts the corrected intensity of the first burst has to be multiplied by the number of following bursts. ${ }^{61}$ In ref. 64 it is even discussed that particularly when the dead time of a high-intensity signal influences the following mass peaks, a dead time correction becomes almost impossible. However, owing to the decreased saturation problems, the intensity of minority isotope peaks can be summed over the entire bursts. The determination of accurate diffusion profiles therefore implies separation of the calculation into different parts, since the ${ }^{18} \mathrm{O}$ fraction may vary from almost unity to natural abundance. Consequently, accurate analysis of ${ }^{18} \mathrm{O}$ diffusion profiles in the "BA-Burst" mode requires a complicated calculation of ${ }^{18} \mathrm{O}$ fractions. Compared to the HCBU and the BA mode, the conventional "Collimated" mode for imaging can show an enhanced performance in terms of higher lateral resolution and accuracy due to the reduced primary ion intensity (not shown). However, particularly the modified beam guidance in the CBA mode enables essential advantages. This is discussed in the following section.

\section{The novel "Collimated Burst Alignment" mode}

\section{Beam guidance of the CBA mode}

The "Collimated Burst Alignment" (CBA) mode is a novel primary ion gun mode optimized to accurately determine oxygen isotopic fractions and to enable a significantly improved lateral resolution. In the CBA mode all three ion optical lenses are energized. Compared to the conventional BA mode a higher voltage is applied to the "Lens Source" in order to diverge the bismuth beam. Aperture 1 blanks out bismuth ions deviating from the optical axis whereas the collimated near-axis ions pass. Hence, increasing the "Lens Source" voltage collimates the primary ion beam and decreases the primary ion intensity in a first step. Owing to the collimation, however, the beam crossover shifts from aperture 2 towards the target. A high voltage applied to "Lens Magnification" repositions the beam crossover in aperture 2. The current measurements (scope 1 and 2) on aperture 1 and 2 show the actual beam position. Moreover, a first step of focusing can be done by aligning "Lens Magnification" in such a way that the current signal on aperture 2 (scope 2) achieves a rectangular-like shape. Since the ion beam is focused on and oscillating over aperture 2 during the current measurement, a rectangular-like current signal indicates a small primary ion beam spot size, at least at aperture 2. A second step of focusing is done by adjusting the "Lens Target" and "X/Y Stigmator", all the same as in the BA mode alignment.

Having the beam crossover position in aperture 2 supports a time-effective alignment and focusing of the primary ion beam due to in situ spot size information. Therefore, the CBA mode enables a simple performance tuning in terms of either higher lateral resolution or secondary ion yield. Compared to the "Collimated" mode the CBA mode is also supposed to show higher performance in terms of increased mass resolution when using the burster, see ref. 65. A detailed description of the CBA alignment procedure is given in the Appendix.

\section{Lateral resolution of the CBA mode}

Secondary Ion Mass Spectrometry (SIMS) not only enables a detailed surface chemical analysis but also a nano-scaled 
chemical imaging. A critical review on "SIMS imaging of the nanoworld" is given by Senoner and Unger. ${ }^{71}$ In the surface analysis community different approaches for the quantification of image resolution are used. In the commonly applied "knife edge method", for example, an image over a straight edge is evaluated. But the latter determines the sharpness of an image and differs from the original meaning of lateral resolution. A very well defined procedure to determine the lateral resolution is suggested by Senoner, Wirth and Unger and is based on the separation of stripes. ${ }^{69,72}$ Therefore, we measured the lateral resolution of the CBA mode on the certified reference material BAM-L200 (ref. 68) by imaging well defined 3-stripe-gratings. The reference material includes several parts, each consisting of two AlGaAs stripes separated by one GaAs stripe, all of the same width, see Fig. 4c. As a reasonable resolution criterion to determine whether an image profile of a grating is resolved, a signal-to-noise ratio $D / \sigma_{\mathrm{NR}} \geq 4$ is suggested in ref. 69. Fig. $4 \mathrm{a}$ and $\mathrm{b}$ show ToF-SIMS image line scan $\left(\mathrm{Al}^{+}\right)$profiles in the CBA mode $\left(\mathrm{Bi}_{1}{ }^{+}, \mathrm{Bi}_{3}{ }^{++}\right)$obtained from the BAM-L200 reference sample. The calculated signal-to-noise ratios between 4.3 for $\mathrm{Bi}_{1}^{+}$and 5.4 for $\mathrm{Bi}_{3}^{++}$are in agreement with the resolution
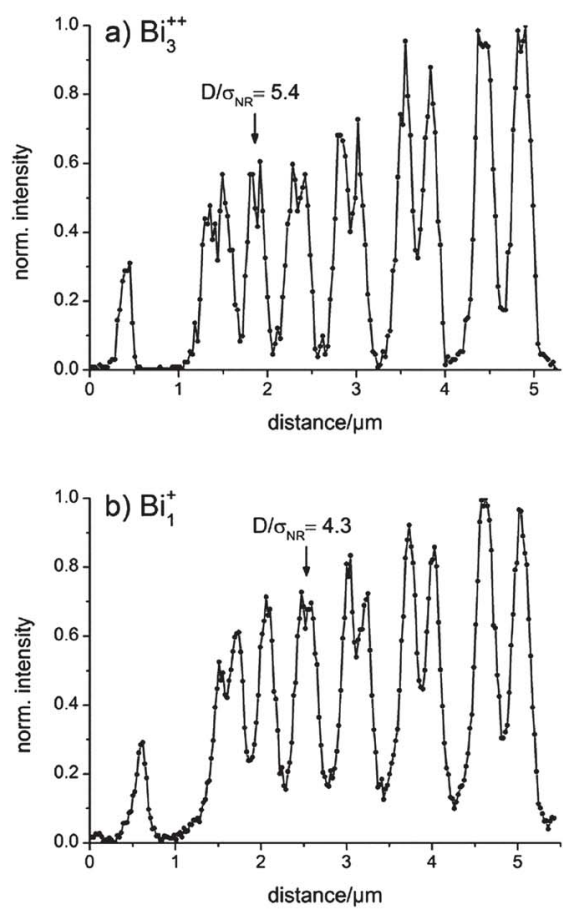

c)

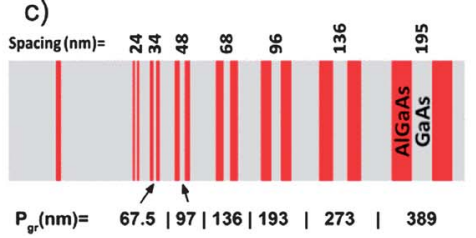

Fig. 4 Profiles resulting after summing 100 scans $\left(\mathrm{Al}^{+}\right)$of the certified BAM-L200 reference material in the CBA mode using (a) $\mathrm{Bi}_{3}{ }^{++}$and (b) $\mathrm{Bi}_{1}{ }^{+}$primary ion clusters. A grating period $P_{\mathrm{gr}}$ of $97 \mathrm{~nm}$ (a) and $136 \mathrm{~nm}$ (b) could be resolved laterally according to the criterion in ref. 69. (c) Scheme of the nano-scale stripe pattern of BAM-L200. criterion; therefore five gratings are resolved in Fig. 4a and four in Fig. 4b. A higher signal-to-noise ratio can be reached by increasing the measurement acquisition time. Owing to instrumental drifts caused by the experimental environment, however, in our measurements a shorter acquisition time had to be chosen. For analysis, the $\mathrm{Al}^{+}$intensity signal of a $7 \times 7 \mu^{2}$ large area was summed over 100 scans. After an optimal primary ion beam alignment, a lateral resolution (l.r.) of $68 \mathrm{~nm}<$ l.r. $<$ $96 \mathrm{~nm}$ for $\mathrm{Bi}_{3}{ }^{++}$and $96 \mathrm{~nm}<$ l.r. $<136 \mathrm{~nm}$ for $\mathrm{Bi}_{1}{ }^{+}$(both $\sim 0.05 \mathrm{pA}$ primary ion current, $75 \mu$ s cycle time) is obtained. The higher lateral resolution obtained for the doubly charged $\mathrm{Bi}_{3}{ }^{++}$ions results from their doubled kinetic energy. With increased kinetic energy the importance of Coulomb repulsion of the primary ions is supposed to decrease; therefore a smaller spot size can be reached. The achievable lateral resolution in ToFSIMS imaging depends on an optimized focus of the primary ion beam and an increased efficiency of desorbed secondary ions. ${ }^{73}$ These properties and further data on the modes considered in this paper are summarized in Table 1.

\section{Analysis of ${ }^{18} \mathrm{O}$ isotope fractions in different modes}

A $\left(\mathrm{La}_{0.6} \mathrm{Sr}_{0.4}\right) \mathrm{CoO}_{3-\delta}$ (LSC) thin layer on YSZ was analyzed to obtain the ${ }^{18} \mathrm{O}$ natural abundance. The primary ion gun was operated in both the conventional BA and the novel CBA mode. For analysis, $\mathrm{Bi}_{1}{ }^{+}$primary ions with an emission current of $1 \mu \mathrm{A}$ were used. Fig. 5 displays the results of 40 analysis cycles for BA and CBA modes. The uncertainty of measurements expressed as a standard deviation is related to only statistical errors. The bar in Fig. 5 represents the natural ${ }^{18} \mathrm{O}$ abundance range of $(2.05 \pm$ $0.14) \times 10^{-3}$ given by the National Institute of Standards and Technology (NIST). The average ${ }^{18} \mathrm{O}$ fraction obtained in the BA mode shows an erroneously high abundance of $2.58 \times 10^{-3}$. Performing analyzes in the CBA mode results in an ${ }^{18} \mathrm{O}$ fraction of $2.07 \times 10^{-3}$. The more accurate determination of the oxygen isotopic fraction is enabled by shifting the secondary ion intensities to an optimal level, with details being given in the following sections. Owing to the low primary ion intensity in the CBA mode the count rates of the major and minor isotopes are decreased. The resulting low count rates for the minor isotope ${ }^{18} \mathrm{O}^{-}$show the greatest impact on the counting statistics. Compared to the standard deviation of $( \pm 0.058) \times 10^{-3}$ obtained in BA mode, therefore, a slightly larger deviation of $( \pm 0.092) \times 10^{-3}$ could be determined in CBA mode.

This raises the question whether the BA mode always leads to erroneous results. As a first optimization of the BA mode, the relatively high primary ion current $\left(\mathrm{Bi}_{1}{ }^{+}, 1 \mathrm{pA}\right.$ pulsed, $20 \mu$ s cycle time) was decreased to reach lower intensities in the ${ }^{16} \mathrm{O}$ major isotope mass peak. In Fig. 6 the resulting ${ }^{18} \mathrm{O}$ fraction is plotted versus the ${ }^{16} \mathrm{O}$ intensity for the $\mathrm{SrTiO}_{3}$ sample. The determined ${ }^{18} \mathrm{O}$ tracer fractions for different operation modes, cluster sizes and primary ion emission currents are also included. Each presented experimental value is the arithmetic means of at least 40 analysis cycles and the standard deviation represents the statistical errors. 
Table 1 Summary of operation parameters and properties of different modes. The symbol * indicates values from the ION-TOF help. Mass resolution values ${ }^{\dagger}$ depend on the chosen primary ion pulse width

\begin{tabular}{|c|c|c|c|c|c|}
\hline Operation mode & $\begin{array}{l}\text { HCBU (High Current } \\
\text { Bunched) }\end{array}$ & BA (Burst Alignment) & Collimated & \multicolumn{2}{|c|}{ CBA (Collimated Burst Alignment) } \\
\hline Lateral resolution (1.r.) & $\sim 2$ to $10 \mu \mathrm{m}^{*}$ & $\sim 250$ to $300 \mathrm{~nm}^{*}$ & $\sim 100 \mathrm{~nm}^{*}$ & $\mathrm{Bi}_{1}^{+}: 96<$ 1.r. $<136 \mathrm{~nm}$ & $\mathrm{Bi}_{3}{ }^{++}: 68<$ l.r. $<96 \mathrm{~nm}$ \\
\hline Activated burster & - & $>6000^{\dagger}$ & $>6000^{\dagger}$ & $>6000$ & $>6000$ \\
\hline DC-current & $16 \mathrm{nA}$ & $0.4-0.75 \mathrm{nA}$ & $50 \mathrm{pA}$ & $70 \mathrm{pA}$ & $210 \mathrm{pA}$ \\
\hline Lens Source & $\sim 3150 \mathrm{~V}$ & $\sim 3300 \mathrm{~V}$ & $\sim 3900 \mathrm{~V}$ & $\sim 3750 \mathrm{~V}$ & $\sim 3500 \mathrm{~V}$ \\
\hline
\end{tabular}

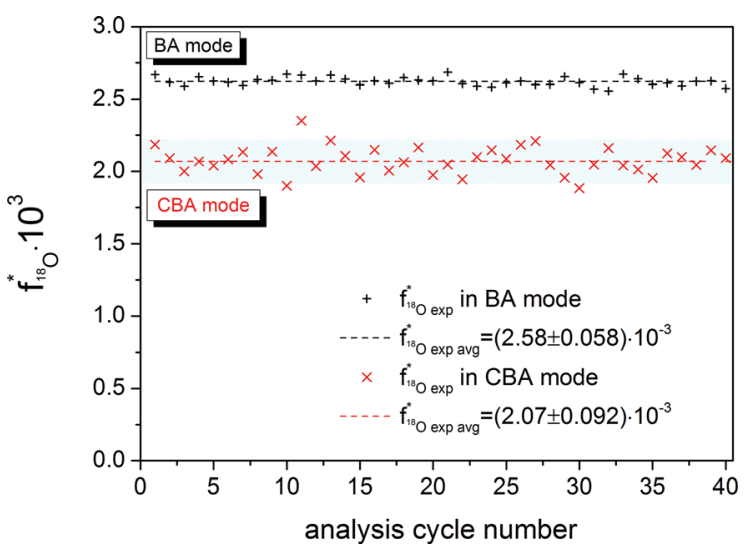

Fig. $5{ }^{18} \mathrm{O}$ fraction in a $\left(\mathrm{La}_{0.6} \mathrm{Sr}_{0.4}\right) \mathrm{CoO}_{3-\delta}$ (LSC) thin film obtained using $\mathrm{Bi}_{1}{ }^{+}$ primary ions and $1 \mu \mathrm{A}$ emission current operating the primary ion gun in both the $\mathrm{BA}$ and the CBA mode. The bar represents the NIST values given for the ${ }^{18} \mathrm{O}$ natural abundance. The dashed lines show the arithmetic mean of the ${ }^{18} \mathrm{O}$ fraction of 40 analysis cycles. After PLD preparation, the LSC layer was annealed in an ambient atmosphere for $30 \mathrm{~min}$ at $600^{\circ} \mathrm{C}$.

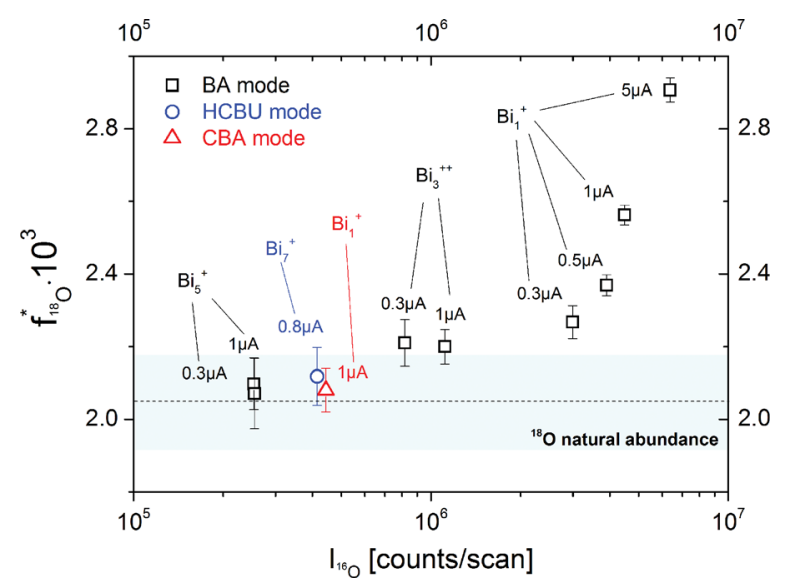

Fig. $6{ }^{18} \mathrm{O}$ fractions in an as-received $\mathrm{SrTiO}_{3}$ single crystal obtained for different operation modes (BA, HCBU, CBA), bismuth emission currents $(0.3$ to $5 \mu \mathrm{A})$ and bismuth cluster sizes $\left(\mathrm{Bi}_{1}{ }^{+}, \mathrm{Bi}_{3}{ }^{++}, \mathrm{Bi}_{5}{ }^{+}, \mathrm{Bi}_{7}{ }^{+}\right)$. The bar represents the NIST values given for the ${ }^{18} \mathrm{O}$ natural abundance.

A clear correlation between the ${ }^{16} \mathrm{O}$ intensity and the detected ${ }^{18} \mathrm{O}$ tracer fraction was found. The higher the major isotope intensity $I_{160}$ the larger is the deviation from the correct ${ }^{18} \mathrm{O}$ fraction. Operating the BA mode with the lowest stable emission current (in our ToF-SIMS setup $0.3 \mu \mathrm{A}$ ) results in an ${ }^{18} \mathrm{O}$ fraction of $2.27 \times 10^{-3}$ compared to $2.56 \times 10^{-3}$ at $1.0 \mu \mathrm{A}$. However, despite lowering the emission current to a minimum, the natural ${ }^{18} \mathrm{O}$ abundance value is not reached. This can only be achieved when using low intensive clusters in $\mathrm{BA}$ mode $\left(\mathrm{Bi}_{5}^{+}\right)$or HCBU mode $\left(\mathrm{Bi}_{7}^{+}\right)$.

The measurement in CBA mode, on the other hand, results in an oxygen isotopic fraction of $2.08 \times 10^{-3}$ and thus leads to an accurate value even though a high emission current $(1 \mu \mathrm{A})$ and $\mathrm{Bi}_{1}{ }^{+}$primary ions were used. This is attributed to the fact that in CBA mode still low secondary ion intensities are reached, even when $\mathrm{Bi}_{1}{ }^{+}$primary ions are used. Accordingly, the ${ }^{18} \mathrm{O}$ fraction could be accurately determined without limitations of emission current or cluster sizes.

In Fig. 7 the oxygen isotopic fractions are displayed for a $\left(\mathrm{La}_{0.6} \mathrm{Sr}_{0.4}\right) \mathrm{FeO}_{3-\delta}(\mathrm{LSF})$ thin layer on $(\mathrm{Zr}, \mathrm{Y}) \mathrm{O}_{2-\delta}(\mathrm{YSZ})$ analyzed immediately after PLD preparation (without annealing in ambient air). All general trends observed above $\left(\mathrm{SrTiO}_{3}\right.$, Fig. 6) are found again. As long as ${ }^{16} \mathrm{O}$ intensities are very high, ${ }^{18} \mathrm{O}$ tracer fractions are systematically too high. Irrespective of the

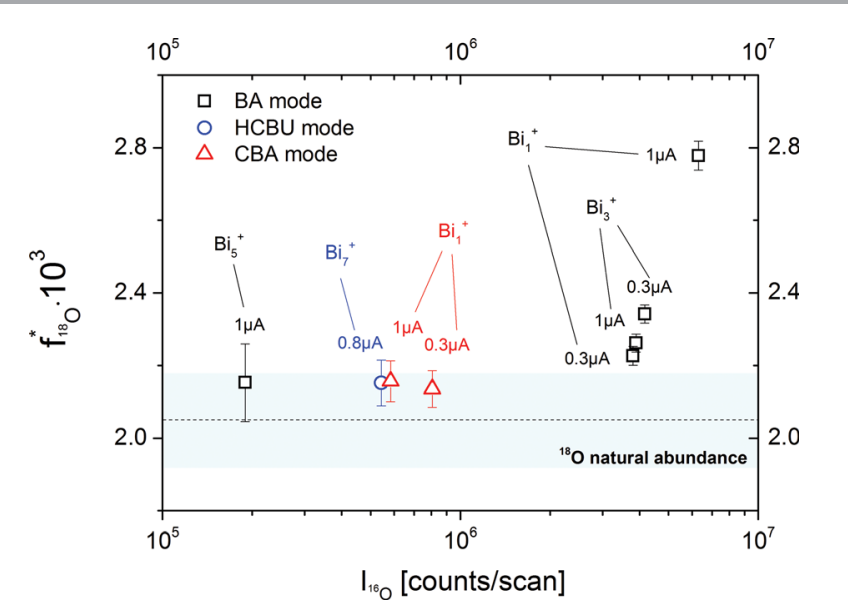

Fig. 7 Dependency of the ${ }^{18} \mathrm{O}$ fraction in a $\left(\mathrm{La}_{0.6} \mathrm{Sr}_{0.4}\right) \mathrm{FeO}_{3-\delta}(\mathrm{LSF})$ thin layer for different operation modes ( $C B A, B A, H C B U)$, bismuth emission currents (0.3 to $1 \mu \mathrm{A})$ and bismuth cluster sizes $\left(\mathrm{Bi}_{1}{ }^{+}, \mathrm{Bi}_{3}{ }^{+}, \mathrm{Bi}_{5}{ }^{+}, \mathrm{Bi}_{7}{ }^{+}\right)$. The sample was investigated immediately after preparation by pulsed laser deposition. A higher ${ }^{18} \mathrm{O}$ fraction of $2.16 \times 10^{-3}$ was obtained due to higher ${ }^{18} \mathrm{O}_{2}$ amount in the ${ }^{16} \mathrm{O}_{2}$ gas bottle (cf. ref. 54). 


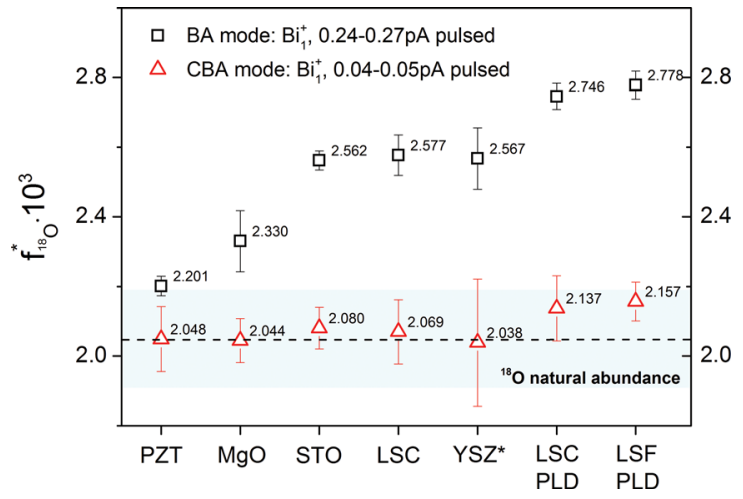

Fig. $8{ }^{18} \mathrm{O}$ fractions measured on $\mathrm{Pb}(\mathrm{Zr}, \mathrm{Ti}) \mathrm{O}_{3}$ (PZT), $\mathrm{MgO}, \mathrm{SrTiO}_{3}(\mathrm{STO}),(\mathrm{La}, \mathrm{Sr})$ $\mathrm{CoO}_{3-\delta}(\mathrm{LSC}), \mathrm{YSZ},(\mathrm{La}, \mathrm{Sr}) \mathrm{FeO}_{3-\delta}(\mathrm{LSF})$ in both the "Burst Alignment" (BA) and the "Collimated Burst Alignment" (CBA) operation mode. The ${ }^{18} \mathrm{O}$ fraction on YSZ* was measured with very low count rate, therefore the standard deviation increased due to counting statistics. PLD indicates thin films measured before annealing. The bar represents the values given by NIST for the natural ${ }^{18} \mathrm{O}$ abundance.

mode, low $I_{160}$ values yield correct values but only the CBA mode can achieve the correct results with all Bi clusters.

Interestingly, the results obtained at low $I_{160}$ also show a slightly enhanced ${ }^{18} \mathrm{O}$ tracer fraction in this case; for the CBA mode $2.16 \times 10^{-3}$ is obtained. Such enhanced values after PLD production were repeatedly found, also for an as-prepared LSC layer a fraction of $2.14 \times 10^{-3}$ was determined. Therefore, annealing for $30 \mathrm{~min}$ at $600{ }^{\circ} \mathrm{C}$ in ambient air was performed and caused a decrease to $2.07 \times 10^{-3}$ and thus to the correct natural abundance value. These results can be understood from the fact that the ${ }^{18} \mathrm{O}$ fraction in bottled (pure) oxygen is often not at the natural abundance level; values between $2.2 \times 10^{-3}$ and $5.2 \times 10^{-3}$ are reported in ref. 54. When using such $\mathrm{O}_{2}$ during PLD layer preparation, a higher ${ }^{18} \mathrm{O}$ fraction results in the samples but subsequent annealing in ambient air leads to a complete oxygen exchange due to the high diffusion coefficients $^{27}$ and thus to the natural abundance value.

Fig. 8 presents experimental data on the ${ }^{18} \mathrm{O}$ fraction in several oxides measured by operating ToF-SIMS in both the BA and the CBA mode. The ordinate corresponds to the measured oxygen fraction given in percent and the abscissa assigns the analyzed oxides ordered from the left to the right in terms of increasing sputter yield. ${ }^{18} \mathrm{O}$ "natural abundance" values obtained in the BA mode consistently show erroneously high ${ }^{18} \mathrm{O}$ fractions, whereas analysis in the CBA mode enables an accurate determination. The higher the sputter yield, the higher is the error of the determined oxygen isotopic fractions. These results also show that both the high accuracy for all Bi clusters and the optimized lateral resolution result from low SI intensities and can only be reached due to the modified beam guidance in CBA mode.

\section{Discussion of the improved accuracy in CBA mode}

Fig. 9 displays a typical example how the dead time affects the major isotope $\left({ }^{16} \mathrm{O}\right)$ mass peak. The mass spectrum shows the
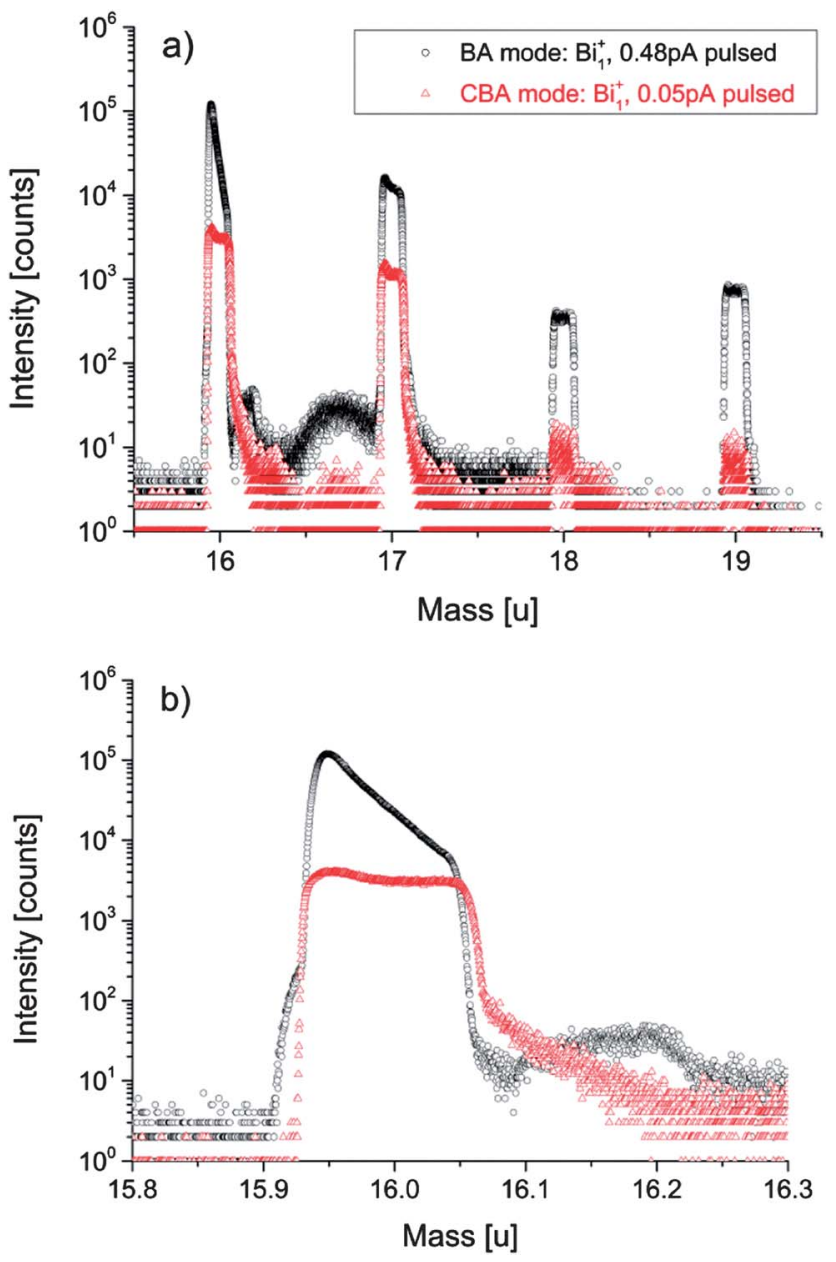

Fig. 9 Negative secondary ion mass spectrum including all oxygen isotopes (a) and at a magnification around $16 \mathrm{u}$ (b) obtained in the "Burst Alignment" (BA) and the "Collimated Burst Alignment" (CBA) for a $\mathrm{SrTiO}_{3}$ sample. The BA mass spectrum shows the dead time effect on the signal intensity in addition to preceding ions and collision-induced delayed ions. The significantly decreased intensities in CBA mode reduce the dead time effect and ion interactions. The peaks around $17 \mathrm{u}$ and $19 \mathrm{u}$ are mainly caused by ${ }^{16} \mathrm{OH}^{-}$and $\mathrm{F}^{-},{ }^{18} \mathrm{OH}^{-}$, respectively.

part around the oxygen isotopes in a negative secondary ion mass spectrum and reveals very significant differences in secondary ion signals between the BA and the CBA mode. In the $\mathrm{BA}$ mode the ${ }^{18} \mathrm{O}$ mass peak is not significantly affected by saturation due to dead time. The ${ }^{16} \mathrm{O}$ peak, however, exhibits a shoulder at lower masses which suggests that some ions arrive at the detector earlier than the main part of the secondary ions. We suppose that the primary ion pulse deviates from an ideal rectangular pulse shape due to Coulomb repulsion in the highly intensive primary ion beam. Both noise signal and preceding ions can already saturate the detector which erroneously causes a lower intensity maximum. The following main peak of the major isotope ${ }^{16} \mathrm{O}$ in $\mathrm{BA}$ mode strongly saturates the single ion counting detector. The latter is not able to recover from the huge amount of secondary ions per primary ion pulse before the next analysis cycle starts. Therefore channel by channel lower secondary ion intensities are detected. The pronounced dead 
time effect gets visible as a steep intensity descent during the entire major isotope mass peak. In such a case of very high intensities the Poissonian correction is supposed to be not sufficient to recalculate the true number of ions impinging the detector and the analysis results in underestimated ${ }^{16} \mathrm{O}$ intensity.

A very broad and intensive background signal between $16 \mathrm{u}$ and $17 \mathrm{u}$ follows the major isotope mass peak. The increase of the ion counts between the masses $16.09 \mathrm{u}$ and $16.23 \mathrm{u}$ indicates that the detector recovers from the previous saturation. However, intensities are so high that another saturation of the detector sets in. This is visualized by the steep intensity descent, followed by increasing counts due to renewed recovering of the detector. Two possible explanations of the background signal between $16 \mathrm{u}$ and $17 \mathrm{u}$ are offered in the literature: in ref. 58 the background signal is ascribed to electrons disturbing the negative mass spectra; these electrons partially result from charge compensation or they are generated from primary ion and secondary ion interactions with various surfaces within the instrument. Analysis in ref. 61 suggest that the background signal is due to ion collisions in the analyzer caused by the huge number of ${ }^{16} \mathrm{O}$ ions.

In accordance with the latter interpretation we assume that due to their collisions ${ }^{16} \mathrm{O}$ ions could be detected as delayed or do not arrive at the detector at all. As a consequence of the underestimated ${ }^{16} \mathrm{O}$ signal, the calculated ${ }^{18} \mathrm{O}$ fraction erroneously shifts to a higher value. Owing to preceding ions, ions of the following masses $17 \mathrm{u}$ and $18 \mathrm{u}$ could be affected by dead time effects even before the corresponding secondary ions arrive at the detector. Since the dead time probability function is not precisely known and the dead time affects the following nominal masses, a Poissonian correction becomes almost impossible. This further affects the calculated ${ }^{18} \mathrm{O}$ fraction.

In the CBA mode, primary ion currents are adjusted in order to reduce effects caused by the dead time and ion interactions. The corresponding colored mass spectrum in Fig. 9 shows a decreased intensity for both major and minor isotopes. Preceding the ${ }^{16} \mathrm{O}$ mass peak a very low noise signal is detected. The steeply rising slope indicates the first ${ }^{16} \mathrm{O}$ ions arriving at the detector. The detector gets slightly saturated, which is indicated by the small decrease of the signal. However, after a time interval of $\sim 20$ ns detection recovers (see Fig. $9 b$ ), resulting in an intensity plateau. The chosen integration interval, which corresponds to the dead time $\tau=65.4 \mathrm{~ns}$, sums the preceding counts as well as the counts of the entire mass peak. Because of low intensity saturation, Poissonian statistics is now suitable to correct the experimentally obtained counts. Since preceding ions are included in the integration interval, a possible saturation of the first part in the mass peak of interest is taken account of Poissonian correction.

Even if a low primary ion current in CBA mode minimizes ion interactions there are still decelerated ions following the major isotope mass peak. However, in contrast to the broad background peak in the BA mode there is no dead time effect observable in the low intensity background signal of the CBA mode. As a result, a more accurate oxygen isotopic fraction can be determined in addition to an improved lateral resolution.
The performance of the CBA mode is discussed in more detail in ref. 65 featuring different measurement techniques and application examples. There, also more information on the still high mass resolution of the CBA mode is given, which would, for example, allow a separation of $\mathrm{H}_{2}{ }^{16} \mathrm{O}$ and ${ }^{18} \mathrm{O}$.

\section{Conclusions}

The operation of established ToF-SIMS modes often results in high primary ion and secondary ion intensities. Pronounced dead time effects and ion interactions can lead to an underestimated value of ${ }^{16} \mathrm{O}$ counts due to saturation of the major isotope mass peak. Consequently, the determination of oxygen isotopic fractions is affected in such a way that the calculated ${ }^{18} \mathrm{O}$ fraction shifts to a higher value. Analysis in BA and HCBU modes therefore shows an erroneously high ${ }^{18} \mathrm{O}$ fraction except for very low primary ion currents resulting when using the largest bismuth clusters $\left(\mathrm{Bi}_{5}{ }^{+}, \mathrm{Bi}_{7}{ }^{+}\right)$. Applying the novel CBA mode enables determination of accurate oxygen isotopic fractions irrespective of the chosen primary ion currents and cluster sizes. Owing to the lower primary ion intensity, a further advantage of the CBA mode is a significantly optimized lateral resolution. Operating CBA with $\mathrm{Bi}_{1}{ }^{+}$and $\mathrm{Bi}_{3}{ }^{++}$clusters enables a lateral resolution of sub- $100 \mathrm{~nm}$. Owing to the modified primary ion beam guidance also the mass resolution can be increased. Moreover, a simple tuning is possible for either achieving higher lateral resolution or an improved detection limit.

\section{Appendix: how to align the CBA mode}

The following instructions detail the alignment procedure of the CBA mode when operating a bismuth ion gun on a TOF. SIMS 5 machine manufactured by ION-TOF. The fundamental idea of the CBA mode, however, can be applied to all ion guns with at least three main ion lenses. Although the same general tendencies can be expected, the exact changes in lateral resolution or intensity will depend on the individual ion gun setup.

Based on an already aligned BA mode, we activated the DC mode and used the "Faraday Cup" to run a target current measurement (manual) in positive mode with illumination turned off. The voltage applied on "Lens Source" has to be increased to $\sim 3500$ to $4000 \mathrm{~V}$ in order to collimate the primary ion beam. While increasing "Lens Source" voltage, the "Direct Current" (DC) is observed to decrease simultaneously. $\mathrm{If}^{\mathrm{Bi}_{1}}{ }^{+}$ ions are used for analysis, a good "Lens Source" voltage is aligned when a target current of $\sim 140 \mathrm{pA}$ is reached. Owing to the voltage increase, the ion beam can shift out of the previously aligned center in the BA mode. In order to measure the correct DC current, the beam should be re-centered using the scope function in apertures 1 and 2 . The upper part of the beam is aligned when scope 1 and 2 signals are centered as visualized in Fig. 10a and b. Then, the scope function is stopped to recenter the near-target part of the beam by optimizing " $\mathrm{X} / \mathrm{Y}$ Blanking". "X/Y Blanking" voltages are adjusted when the target current (DC) reaches its maximum. When the first alignment procedure is finished, an additional adjustment cycle (like increasing "Lens Source" voltage and re-centering of 


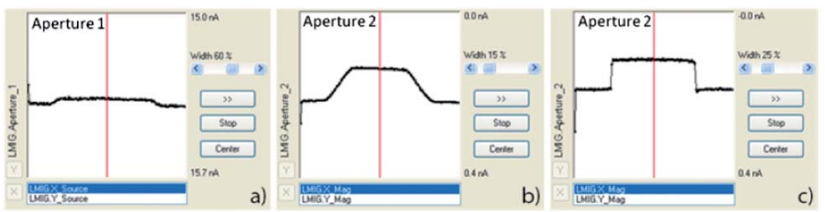

Fig. 10 Primary ion current measurements on primary ion gun aperture 1 (scope 1) and aperture 2 (scope 2) of an already aligned ion beam. (a) Current signal on aperture 1 after primary ion beam collimation with already decreased intensity. (b) Current signal on aperture 2 after collimation without any voltage applied to "Lens Magnification". (c) Current signal on aperture 2 shows a rectangular shape after an optimal voltage was applied to "Lens Magnification".

the upper and near-target beam) would be necessary to finally reach a target current of $\sim 140 \mathrm{pA}$. The current signal in scope 1 of aperture 1 should now show a decreased amplitude like that displayed in Fig. 10a.

As a next important step, the voltage on "Lens Magnification" is gradually increased from zero to approximately 12-13 $\mathrm{kV}$ while observing the current signal of scope 2 approaching a rectangular-like shape. The optimal voltage is reached when the steepness of the rectangular-like current signal reaches its maximum, see Fig. 10c. The upper and near-target parts of the beam have to be re-centered again. The DC current is expected to be approximately $70 \mathrm{pA}$, otherwise "Lens Source" and "Lens Magnification" voltages can be fine-tuned. The primary ion gun is aligned to a DC current of $\sim 70 \mathrm{pA}$ optimized for oxygen isotopic measurements using $\mathrm{Bi}_{1}{ }^{+}$. Since bismuth clusters like $\mathrm{Bi}_{3}{ }^{++}$have a lower relative intensity in the DC beam than $\mathrm{Bi}_{1}{ }^{+}$, a higher DC current has to be aligned to finally reach a pulsed primary ion beam current of $\sim 0.05 \mathrm{pA}$ (20 $\mu$ s cycle time). The relative primary ion currents of several Bi clusters in the DC beam are given in ref. 74 .

The following fine-focusing steps are monitored by observing a "Secondary Electron" (SE) image on the "A-Grid" (chopper grid, $100 \mu \mathrm{m}$ spacing) with a "Field of View" (FoV) of at least $30 \times 30 \mu^{2}$, the reference height is already adjusted. When focusing the beam for the first time in the CBA mode, the "X/Y Stigmator" should be set to zero before the "Lens Target" is optimized for the best focus. Adjusting the "X/Y Stigmator" will compensate possible astigmatism of the beam resulting in a better resolution. After successful focusing, the SE image is expected to well-resolve at least $10 \times 10 \mu \mathrm{m}^{2}$ (FoV). When the primary ion beam is aligned and focused once, a setting file should be saved. For further beam alignment it is just necessary to load the CBA setting file, to maximize the DC current by optimizing "X/Y Blanking” and fine-focusing as in the BA mode.

The higher the desired lateral resolution the lower has the primary ion current to be adjusted. The minimum limit, however, is exceeded when the amplitude in the scope 1 signal disappears and therefore an alignment of the ion beam is impossible. As an optimal value to determine accurate oxygen isotopic fractions, a DC current of $\sim 70 \mathrm{pA}$ for $\mathrm{Bi}_{1}{ }^{+}$and $\sim 210 \mathrm{pA}$ for $\mathrm{Bi}_{3}{ }^{++}(0.03-0.05 \mathrm{pA}$ pulsed at $100 \mu$ s cycle time) is suggested. The voltages and currents applied to modify the CBA mode may vary on other ToF-SIMS instruments, for example, due to different aperture abrasion or primary ion species.

\section{Acknowledgements}

Financial support by Christian Doppler Laboratory of Ferroic Materials and Austrian Science Fund (FWF) project F 4509-N16 is gratefully acknowledged. We also thank Klaus Reichmann, Graz University of Technology, for providing PZT samples and Sandra Kogler for providing LSF samples.

\section{References}

1 S. B. Adler, Chem. Rev., 2004, 104, 4791-4843.

2 W. C. Chueh, Y. Hao, W. Jung and S. M. Haile, Nat. Mater., 2012, 11, 155-161.

3 J. L. M. Rupp, Solid State Ionics, 2012, 207, 1-13.

4 T. Horita, T. Shimonosono, H. Kishimoto, K. Yamaji, M. E. Brito, Y. Hori and H. Yokokawa, Electrochem. Solid State Lett., 2010, 13, B135-B138.

5 J. Mizusaki, K. Amano, S. Yamauchi and K. Fueki, Solid State Ionics, 1987, 22, 313-322.

6 T. Horita, D. Cho, T. Shimonosono, H. Kishimoto, K. Yamaji, M. E. Brito and H. Yokokawa, J. Electrochem. Soc., 2012, 159, F476-F481.

7 J. Mizusaki, K. Amano, S. Yamauchi and K. Fueki, Solid State Ionics, 1987, 22, 323-330.

8 M. Sase, K. Yashiro, K. Sato, J. Mizusaki, T. Kawada, N. Sakai, K. Yamaji, T. Horita and H. Yokokawa, Solid State Ionics, 2008, 178, 1843-1852.

9 M. Sase, F. Hermes, K. Yashiro, K. Sato, J. Mizusaki, T. Kawada, N. Sakai and H. Yokokawa, J. Electrochem. Soc., 2008, 155, B793-B797.

10 A. Chroneos, B. Yildiz, A. Tarancon, D. Parfitt and J. A. Kilner, Energy Environ. Sci., 2011, 4, 2774-2789.

11 I. D. Seymour, A. Tarancon, A. Chroneos, D. Parfitt, J. A. Kilner and R. W. Grimes, Solid State Ionics, 2012, 216, 41-43.

12 E. Mutoro, S. Gunther, B. Luerssen, I. Valov and J. Janek, Solid State Ionics, 2008, 179, 1835-1848.

13 S. Fearn, J. C. H. Rossiny, J. A. Kilner and J. R. G. Evans, Solid State Ionics, 2012, 211, 51-57.

14 N. Sakai, T. Horita, K. Yamaji, M. E. Brito, H. Yokokawa, A. Kawakami, S. Matsuoka, N. Watanabe and A. Ueno, J. Electrochem. Soc., 2006, 153, A621-A625.

15 D. P. Rupasov, A. V. Berenov, J. A. Kilner, S. Y. Istomin and E. V. Antipov, Solid State Ionics, 2011, 197, 18-24.

16 Z. Cai, M. Kubicek, J. Fleig and B. Yildiz, Chem. Mater., 2012, 24, 1116-1127.

17 R. Hancke, S. Fearn, J. A. Kilner and R. Haugsrud, Phys. Chem. Chem. Phys., 2012, 14, 13971-13978.

18 J. Janek, B. Luerssen, E. Mutoro, H. Fischer and S. Gunther, Top. Catal., 2007, 44, 399-407.

19 M. Kubicek, A. Limbeck, T. Frömling, H. Hutter and J. Fleig, J. Electrochem. Soc., 2011, 158, B727-B734.

20 R. A. De Souza, M. J. Pietrowski, U. Anselmi-Tamburini, S. Kim, Z. A. Munir and M. Martin, Phys. Chem. Chem. Phys., 2008, 10, 2067-2072.

21 N. J. Simrick, A. Bieberle-Hutter, T. M. Ryll, J. A. Kilner, A. Atkinson and J. L. M. Rupp, Solid State Ionics, 2012, 206, 7-16. 
22 H. Aydin, C. Korte, M. Rohnke and J. Janek, Phys. Chem. Chem. Phys., 2013, 15, 1944-1955.

23 J. L. Hertz and H. L. Tuller, Solid State Ionics, 2007, 178, 915923.

24 M. Burriel, J. Pena-Martinez, R. J. Chater, S. Fearn, A. V. Berenov, S. J. Skinner and J. A. Kilner, Chem. Mater., 2012, 24, 613-621.

25 A. K. Opitz and J. Fleig, Solid State Ionics, 2010, 181, 684-693.

26 A. Opitz, A. Schintlmeister, H. Hutter and J. Fleig, Phys. Chem. Chem. Phys., 2010, 12, 12734-12745.

27 M. Kubicek, Z. Cai, W. Ma, B. Yildiz, H. Hutter and J. Fleig, ACS Nano, 2013, 7, 3276-3286.

28 M. Gerstl, T. Frömling, A. Schintlmeister, H. Hutter and J. Fleig, Solid State Ionics, 2011, 184, 23-26.

29 R. A. De Souza, J. A. Kilner and J. F. Walker, Mater. Lett., 2000, 43, 43-52.

30 M. Y. Oh, A. Unemoto, K. Amezawa and T. Kawada, J. Electrochem. Soc., 2012, 159, F659-F664.

31 V. B. Vert, J. M. Serra, J. A. Kilner and M. Burriel, J. Power Sources, 2012, 213, 270-274.

32 E. N. Coker, J. A. Ohlhausen, A. Ambrosini and J. E. Miller, J. Mater. Chem., 2012, 22, 6726.

33 D. Pergolesi, E. Fabbri, S. N. Cook, V. Roddatis, E. Traversa and J. A. Kilner, ACS Nano, 2012, 6, 10524-10534.

34 G. Y. Yang, G. D. Lian, E. C. Dickey, C. A. Randall, D. E. Barber, P. Pinceloup, M. A. Henderson, R. A. Hill, J. J. Beeson and D. J. Skamser, J. Appl. Phys., 2004, 96, 7500-7508.

35 H. M. Duiker, P. D. Beale, J. F. Scott, C. A. P. Dearaujo, B. M. Melnick, J. D. Cuchiaro and L. D. McMillan, J. Appl. Phys., 1990, 68, 5783-5791.

36 M. F. Zhang, Y. Wang, K. F. Wang, J. S. Zhu and J. M. Liu, J. Appl. Phys., 2009, 105.

37 R. Waser, T. Baiatu and K. H. Hardtl, J. Am. Ceram. Soc., 1990, 73, 1645-1653.

38 S. Rodewald, J. Fleig and J. Maier, J. Am. Ceram. Soc., 2000, 83, 1969-1976.

39 N. J. Donnelly and C. A. Randall, J. Appl. Phys., 2011, 109.

40 N. J. Donnelly and C. A. Randall, J. Am. Ceram. Soc., 2009, 92, 405-410.

41 N. Balke, D. C. Lupascu, T. Granzow and J. Rodel, J. Am. Ceram. Soc., 2007, 90, 1081-1087.

42 R.-A. Eichel, Phys. Chem. Chem. Phys., 2011, 13, 368-384.

43 R. A. De Souza, V. Metlenko, D. Park and T. E. Weirich, Phys. Rev. B: Condens. Matter Mater. Phys., 2012, 85.

44 N. J. Donnelly and C. A. Randall, Appl. Phys. Lett., 2010, 96.

45 J. Claus, M. Leohardt and J. Maier, J. Phys. Chem. Solids, 2000, 61, 1199-1207.

46 T. Frömling, A. Schintlmeister, H. Hutter and J. Fleig, J. Am. Ceram. Soc., 2011, 94, 1173-1181.

47 T. Frömling, H. Hutter and J. Fleig, J. Am. Ceram. Soc., 2012, 95, 1692-1700.
48 K. Reichmann, E. Volkl, M. Ahrens, J. Fleig and J. Votsch, J. Mater. Sci., 2010, 45, 1473-1477.

49 L. Andrejs and J. Fleig, J. Eur. Ceram. Soc., 2013, 33, 779-794.

50 E. Völkl, P. Hillebrand and J. Fleig, J. Electroceram., 2011, 27, 66.

51 J. A. Kilner, S. J. Skinner and H. H. Brongersma, J. Solid State Electrochem., 2011, 15, 861-876.

52 T. Horita, K. Yamaji, N. Sakai, Y. Xiong, T. Kato, H. Yokokawa and T. Kawada, Appl. Surf. Sci., 2003, 203204, 634-638.

53 R. A. De Souza and M. Martin, Phys. Chem. Chem. Phys., 2008, 10, 2356-2367.

54 R. A. De Souza and R. J. Chater, Solid State Ionics, 2005, 176, 1915-1920.

55 T. Horita, K. Yamaji, N. Sakai, Y. Xiong, H. Yokokawa and T. Kawada, Ionics, 2002, 8, 108-117.

56 A. Benninghoven, Angew. Chem., Int. Ed. Engl., 1994, 33, 1023-1043.

57 A. Benninghoven, Surf. Sci., 1973, 35, 427-457.

58 T. Stephan, Planet. Space Sci., 2001, 49, 859-906.

59 A. J. Fahey and S. Messenger, Int. J. Mass Spectrom., 2001, 208, 227-242.

60 P. Fielitz and G. Borchardt, Solid State Ionics, 2001, 144, 71-80.

61 R. A. De Souza, J. Zehnpfenning, M. Martin and J. Maier, Solid State Ionics, 2005, 176, 1465-1471.

62 J. L. S. Lee, I. S. Gilmore and M. P. Seah, Surf. Interface Anal., 2012, 44, 1-14.

63 M. R. Keenan and P. G. Kotula, Surf. Interface Anal., 2004, 36, 203-212.

64 T. Stephan, J. Zehnpfenning and A. Benninghoven, J. Vac. Sci. Technol., A, 1994, 12, 405-410.

65 M. Kubicek, G. Holzlechner, A. Opitz, S. Larisegger, H. Hutter and J. Fleig, Appl. Surf. Sci., 2013, submitted.

66 B. Hagenhoff, D. van Leyen, E. Niehuis and A. Benninghoven, J. Vac. Sci. Technol., A, 1989, 7, 3056-3064.

67 H. W. Werner and N. Warmoltz, J. Vac. Sci. Technol., A, 1984, 2, 726-731.

68 M. Senoner, T. Wirth, W. Unger, W. Osterle, I. Kaiander, R. L. Sellin and D. Bimberg, Surf. Interface Anal., 2004, 36, 1423-U1429.

69 M. Senoner, T. Wirth and W. Unger, J. Anal. At. Spectrom., 2010, 25, 1440-1452.

70 R. N. S. Sodhi, Analyst, 2004, 129, 483-487.

71 M. Senoner and W. Unger, J. Anal. At. Spectrom., 2012, 27, 1050.

72 PD ISO/TR 19319:2013: Surface chemical analysis, Fundamental approaches to determination of lateral resolution and sharpness in beam-based methods, British Standards Institution, 2013.

73 B. Hagenhoff, Mikrochim. Acta, 2000, 132, 259-271.

74 F. Kollmer, Appl. Surf. Sci., 2004, 231-232, 153-158. 